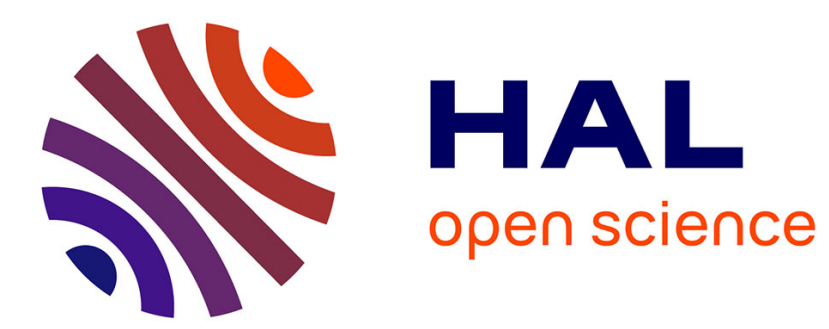

\title{
Méthodes expérimentales de caractérisation des catalyseurs industriels
}

\author{
J.C. Vedrine
}

\section{To cite this version:}

J.C. Vedrine. Méthodes expérimentales de caractérisation des catalyseurs industriels. Revue de Physique Appliquée, 1981, 16 (7), pp.373-386. 10.1051/rphysap:01981001607037300 . jpa-00244930

\section{HAL Id: jpa-00244930 https://hal.science/jpa-00244930}

Submitted on 1 Jan 1981

HAL is a multi-disciplinary open access archive for the deposit and dissemination of scientific research documents, whether they are published or not. The documents may come from teaching and research institutions in France or abroad, or from public or private research centers.
L'archive ouverte pluridisciplinaire HAL, est destinée au dépôt et à la diffusion de documents scientifiques de niveau recherche, publiés ou non, émanant des établissements d'enseignement et de recherche français ou étrangers, des laboratoires publics ou privés. 


\title{
Méthodes expérimentales de caractérisation des catalyseurs industriels (*)
}

\author{
J. C. Vedrine \\ Institut de Recherches sur la Catalyse, C.N.R.S., 2, avenue A.-Einstein, F69626 Villeurbanne Cedex, France
}

(Reçu le 21 novembre 1980, révisé le 18 janvier 1981, accepté le 27 mars 1981)

\begin{abstract}
Résumé. - Dans cette mise au point nous avons cherché à présenter de façon très simplifiée aux scientifiques non initiés la Catalyse et surtout les problèmes expérimentaux qui se posent pour l'étude des processus de catalyse hétérogène. Après un rappel sur le phénomène catalytique et les catalyseurs les plus importants, nous abordons brièvement les réacteurs et l'étude des mécanismes réactionnels et de la cinétique chimique. Nous décrivons ensuite les principales méthodes physiques de caractérisation des catalyseurs en apportant un intérêt particulier à l'étude des surfaces et des mesures dans les conditions proches de la réaction catalytique. Enfin quelques exemples permettent de comprendre la complexité des problèmes et la nécessité d'utiliser conjointement plusieurs techniques physiques pour une approche plus exhaustive des propriétés des catalyseurs.
\end{abstract}

\begin{abstract}
In this review article we have tried to present in a very simple manner " Catalysis and its related phenomena and problems » to scientists who are not familiar in this field. Introduction to catalytic phenomenon, catalytic reactions and reaction mechanisms is presented first, followed by a brief summary of the main catalysts and catalytical reactions with special emphasis to industrial interests. Questions, which still remain in catalysis field to explain catalytic properties, are raised and presented in terms of the knowledge of the catalyst and of the influence of the reagents on it (environment, changes in symmetry and oxidation state, surface modifications etc...). The main physical methods, which are of interest for characterizing catalysts properties, are presented with the main data which may be gained for a better understanding. Some examples of the use of the physical methods are given and analyzed. A special emphasis is presented on the necessity of using several physical methods to assess our knowledge on the catalytic properties of different materials. At last a special interest is brought about the characterization of surface properties and about the use of physical methods in conditions as close as possible to those of the catalytic reactions.
\end{abstract}

Introduction. - On sait que l'on entend sous le terme de Catalyseur toute substance capable de faciliter ou orienter sélectivement une réaction chimique déterminée. Le rôle du Catalyseur est généralement d'accroitre la vitesse de la réaction du fait de la diminution de l'énergie d'activation, $E_{\mathrm{a}}$, sans modification de l'énergie nécessaire imposée par les lois classiques de thermodynamique et nécessaire à la réaction.

Lorsque catalyseurs et réactifs sont miscibles (gaz ou liquides) on a affaire à la Catalyse homogène alors qu'en cas contraire (gaz-solide) il s'agit de Catalyse hétérogène. Dans ce dernier cas pour expliquer que les vitesses de réaction sont comparables à celles observées en catalyse homogène, alors que la probabilité de choc entre molécules de réactifs et surface du catalyseur est bien plus faible (facteur $10^{12}$ ), il a fallu admettre que le catalyseur intervient lui-même

(*) Conférence présentée à la réunion 1980 du groupe français de Spectroscopie Mössbauer à Lyon sur le thème "Surfaces, grains fins, catalyse » [20]. dans la réaction par le phénomène de chimisorption qui, en quelque sorte, active les molécules de réactif. Le catalyseur est restitué en fin de réaction et n'est donc pas consommé.

Deux paramètres sont importants en Catalyse, il s'agit des propriétés suivantes du catalyseur :

1) Son activité : mesurée par le taux de transformation des réactifs en produits de réaction par unité de temps.

2) Sa sélectivité : mesurée par la proportion d'un produit donné par rapport à l'ensemble des produits de réaction.

En d'autres termes un catalyseur facilite et oriente une réaction chimique par accroissement sélectif de la vitesse d'une réaction particulière.

En Catalyse hétérogène on distingue deux principaux mécanismes de réaction. Le mécanisme d'Hinshelwood correspond à une réaction entre deux réactifs adsorbés et le mécanisme de Rideal à une réaction entre un réactif en phase gazeuse ou liquide 
et l'autre réactif adsorbé. Les paramètres importants sont donc l'adsorption, la réaction en elle-même et la désorption des produits de réaction. On est alors venu à la notion de sites d'adsorption et de sites actifs et sélectifs en catalyse. Pour essayer de comprendre le phénomène catalytique ou tout du moins d'expliquer ce qui se passe du fait de l'activation et donc du fait de la formation d'intermédiaires réactionnels de duree de vie variable on a été amené à diversifier la gamme des méthodes expérimentales d'appréhension de la réaction catalytique.

Nous analyserons dans ce texte très brièvement les différentes méthodes physiques permettant d'approcher la réaction (analyse des produits de réaction - détermination des mécanismes réactionnels, etc.), et plus longuement celles qui permettent de caractériser le catalyseur lui-même en présence ou en l'absence de réactifs.

1. Cinétique chimique. - L'objectif des études de la cinétique chimique, c'est-à-dire des vitesses de réaction, est la détermination des lois régissant les réactions catalytiques et donc le mécanisme réactionnel. On définit les ordres partiels $(a, b \ldots)$ par rapport aux réactifs $\left(R_{1}, R_{2}, \ldots\right)$ ou l'ordre global à partir de la vitesse de réaction et selon une loi du type :

$$
\frac{\mathrm{d}|\mathrm{P}|}{\mathrm{d} t}=k_{\mathrm{e}}\left|\mathrm{R}_{1}\right|^{a}\left|\mathbf{R}_{2}\right|^{b} \ldots
$$

où $\mathrm{P}$ représente les produits de réaction et $k_{\mathrm{e}}$ la vitesse spécifique ou constante de vitesse définie par :

$$
k_{\mathrm{e}}=A \mathrm{e}^{-E_{\mathrm{a}} / R T}
$$

où $A$ est le facteur de fréquence des sites actifs, $E_{\mathrm{a}}$ l'énergie d'activation et $R$ la constante des gaz parfaits.

En catalyse hétérogène il faut faire intervenir la concentration en réactifs à l'état adsorbé, concentration qui n'est pas connue. En première approximation et moyennant certaines hypothèses, on utilisera les isothermes suivantes, de Langmuir :

$$
\mathrm{R}_{1 \text { ads }}=\frac{k_{1}\left|\mathrm{R}_{1}\right|}{1+k_{1}\left|\mathrm{R}_{1}\right|},
$$

ou de Freundlich :

$$
\mathbf{R}_{1 \text { ads }}=k_{1}\left|\mathbf{R}_{1}\right|^{p},
$$

et les lois précédentes en remplaçant $R_{1}, R_{2} \ldots$ par $R_{1 \text { ads }}, R_{2 \text { ads }}$, etc...

Sans entrer, bien sûr, dans le détail de ces lois on voit déjà l'importance de la détermination du nombre de sites actifs et du nombre de rotations - ou T.O.N. turn over number - c'est-à-dire du nombre de molécules réagissant sur un site par unité de temps. Les lois cinétiques précédentes permettent d'avoir une idée sur ces nombres, mais aucune information ne peut être obtenue sur la nature même de ces sites.
Les mesures cinétiques ont lieu dans de petits réacteurs qui, au laboratoire, sont généralement en verre avec un volume de 10 à $100 \mathrm{ml}$, et un poids de catalyseur de quelques dizaines à quelques centaines de mg. Les réacteurs sont alimentés par un mélange adéquat de réactifs dans un gaz porteur $\left(\mathrm{N}_{2}, \mathrm{He}\right.$ ou $\left.\mathrm{H}_{2}\right)$ dont les proportions relatives peuvent être modifiées à la convenance. Dans l'industrie, les réacteurs sont métalliques et de capacité beaucoup plus élevée et peuvent opérer sous forte pression. Les problèmes de charge, de débits gazeux, de résistance mécanique à l'écrasement du catalyseur, de température réelle de réaction au niveau du catalyseur sont dans ce dernier cas beaucoup plus cruciaux que dans les conditions du laboratoire. L'extrapolation est difficile et passe souvent par une étude en réacteur intermédiaire dit réacteur pilote.

L'analyse des réactifs qui n'ont pas réagi ainsi que des produits de réaction se fait couramment par chromatographie, en particulier en phase gazeuse. Pour cette analyse une prise d'échantillons est faite par l'intermédiaire d'une boucle branchée sur le circuit et qui peut être, manuellement ou automatiquement, mise en contact avec le gaz porteur et les produits envoyés sur une colonne de chromatographie. Dans l'industrie, le pilotage de la réaction (débits des réactifs, température, etc...) est effectué automatiquement à partir du contrôle automatisé des produits de réaction, ce qui permet de tenir compte des variations de la réaction, par exemple du vieillissement du catalyseur.

Pour les études de cinétique et de mécanismes réactionnels on opère à faible taux de conversion et en vitesse initiale de façon à limiter les réactions secondaires parasites. Pour caractériser un catalyseur d'un point de vue qualités industrielles (rendements élevés, sélectivité appropriée, résistance mécanique, résistance à l'empoisonnement et au vieillissement, etc...) on opérera au contraire à des taux de conversion élevés.

Les lois cinétiques permettent de proposer des mécanismes réactionnels. Souvent les possibilités d'étude de mécanismes à partir de données expérimentales sont nombreuses et l'une des méthodes complémentaires la plus courante pour sérier la solution du problème est l'utilisation de molécules marquées - en particulier avec $\mathrm{D}$ au lieu de $\mathrm{H}$ et ${ }^{13} \mathrm{C}$ (pour $\mathrm{RMN}$ ) ou ${ }^{14} \mathrm{C}$ (radioactif) - permettant de suivre le cheminement de fractions des molécules initiales de réactifs et leur insertion dans les produits finaux. La spectrométrie de masse, la spectroscopie micro-onde et la RMN sont aussi des techniques appropriées pour la caractérisation des produits formés.

Les mesures précédentes permettent de caractériser les propriétés des catalyseurs, en particulier d'activité et de sélectivité. La détermination des mécanismes réactionnels permet de préciser les entités ou espèces intermédiaires mises en jeu et le type de réaction 
concernée. Cependant un grand nombre de problèmes restent inconnus :

- Quelle est l'influence de la coordination des ions de transition actifs, de la symétrie de leur environnement immédiat, de leur degré d'oxydation et du degré de covalence des liaisons métal ligande ? Quelles sont les modifications de ces complexes de coordination en cours de réaction ?

- Quelle est la structure des complexes intermédiaires formés et quelle est leur durée de vie ? Comment l'addition de réactifs modifie-t-elle la structure électronique et l'arrangement géométrique de tels complexes?

- Quelles sont les modifications subies par les réactifs au cours de l'adsorption ? Peut-on suivre la formation des produits de réaction ? Quels sont la composition chimique et l'arrangement atomique vrais à la surface d'un catalyseur ? Sont-ils identiques à ceux du volume et sont-ils modifiés par l'addition de réactifs ou lors de la réaction catalytique ? Quelle importance les procédés de préparation jouent-ils ?

- Quand un élément étranger est ajouté à un matériau, comment s'effectue l'incorporation ? Pour les solides a-t-on une solution solide ou un mélange de phases ? Peut-on avoir ségrégation de phases, en surface par exemple ? Les propriétés électroniques du matériau sont-elles modifiées?

- Quand un catalyseur est supporté a-t-on seulement un effet de dispersion qui accroît le rappoṛt de la surface au volume donc le nombre de sites actifs superficiels ? A-t-on une modification des propriétés du support (transfert électronique, acidité, etc...) ?

- Quel est le rôle des transferts électroniques (phase active - support ou pour un alliage métalmétal) et de l'arrangement géométrique et taille des particules de catalyseurs?

$$
\text { - Etc... }
$$

Pour essayer d'apporter des éléments de réponse à ces questions, on dispose de nombreuses techniques, en particulier physiques. Nous allons dans ce qui suit décrire très brièvement les principales techniques physiques que l'on peut utiliser dans les laboratoires de recherche et les principales informations que l'on peut obtenir en nous limitant aux techniques utilisées pour les catalyseurs réels.

Le phénomène catalytique se produisant à la surface des solides catalytiques mais pouvant être influencé par les propriétés de volume nous distinguerons les techniques de surface de celles de volume. Souvent on est amené à étudier les catalyseurs dans des conditions très éloignées de celles de la réaction catalytique et nous porterons un accent particulier sur cet aspect.

2. Principaux types de catalyseurs et de réactions catalytiques. - L'importance de la catalyse dans l'industrie chimique est représentée dans le tableau I qui rapporte la production aux Etats-Unis en 1976 des 70 premiers produits chimiques en termes de quantités. On constate qu'environ $43 \%$ des quantités
Tableau I. - Tonnages des 70 premiers produits chimiques aux Etats-Unis en 1976 (en millions de tonnes).

[Amount of the 70 first products in USA (1976) in thousand of tons.]

\begin{tabular}{|c|c|}
\hline & Tonnage \\
\hline Procédés non catalvtioules & $\overline{120}$ \\
\hline Procédés catalytiques & 91 \\
\hline - catalyse hétérogène & 77 \\
\hline - catalyse homogène & 14 \\
\hline
\end{tabular}

produites sont obtenues à partir de procédés catalytiques, essentiellement en catalyse hétérogène (77. contre $14 \mathrm{M}$ de $\mathrm{t}$ ). Des $91 \mathrm{M}$ de $\mathrm{t}$ de produits formés à partir de procédés catalytiques, environ $53 \%$ (49 $\mathrm{M}$ de $\mathrm{t}$ ) concernent la catalyse d'oxydation dont 43,5 $\mathrm{M}$ de $\mathrm{t}$ en catalyse hétérogène avec $30 \mathrm{M}$ de $t$ pour l'acide sulfurique et $7 \mathrm{M}$ de t pour l'acide nitrique.

On a reporté dans le tableau II des comparaisons qualitatives entre les procédés catalytiques homogènes en phase liquide, avec des problèmes au niveau de la récupération et du recyclage du catalyseur, et les procédés hétérogènes avec des problèmes de réacteurs à cause des difficultés d'échange thermique et de résistance mécanique à l'écrasement du catalyseur.

Tableau II. - Comparaison entre catalyses homogène et hétérogène.

[Comparison between homogeneous and heterogeneous catalysts properties.]

\begin{tabular}{|c|}
\hline homogène $:-$ catalyseurs souvent plus actifs et \\
plus sélectifs \\
- difficultés de séparation et recy- \\
clage de solvants et du catalyseur \\
- réacteurs plus simples et échanges \\
thermiques plus favorables \\
- problèmes de corrosion et de trans- \\
port de matière \\
hétérogène : - catalyseurs plus stables mais moins \\
actifs et moins sélectifs \\
- séparation plus aisée des produits \\
de réaction et du catalyseur \\
- réacteurs plus complexes avec pro- \\
blème important d'échange ther- \\
mique \\
chaleur de réaction récupérable à \\
un niveau thermique plus élevé.
\end{tabular}

Il est bon de noter qu'à l'échelle industrielle il y a des pertes de catalyseur qui, tout en restant faibles par rapport à la masse totale, représentent des quantités non négligeables par exemple les pertes de $\mathrm{Pt}$ dans l'oxydation de $\mathrm{NH}_{3}$ en NO. Dans d'autres cas il est nécessaire d'effectuer de fréquents remplacements du catalyseur (par exemple dans l'oxydation du 
méthanol en formol) ou bien de coûteuses régénérations (par exemple pour les catalyseurs à base de $\mathrm{Pt}$ utilisés dans les procédés de réformage pour la production d'essence ou d'aromatiques et dont la régénération consiste à brûler les dépôts hydrocarbonés formés qui désactivent le catalyseur), ce qui évidemment accroît les pertes de catalyseur.

En dehors de ces problèmes liés à la récupération ou aux pertes de catalyseurs, le choix d'un procédé industriel sera guidé par des considérations évidentes

Tableau III. - Catalyseurs et réactions catalytiques typiques.

[Typical catalysts and catalytic reactions.]

\begin{tabular}{ll}
\multicolumn{1}{c}{ Classe } & \multicolumn{1}{c}{ Réactions catalytiques } \\
Métaux & $\begin{array}{l}\text { oxydation ménagée } \\
\text { oxydation totale } \\
\text { hydrogénation, déshydrogénation } \\
\text { hydrogénolyse... } \\
\text { oxydation ménagée } \\
\text { oxydation totale } \\
\text { déshydrogénation } \\
\text { désulfuration } \\
\text { dénitrification } \\
\text { synthèse d'alcools } \\
\text { Oxydes ou sulfures } \\
\text { demi-conducteurs) }\end{array}$ \\
& isomérisation alcools \\
Oxydes isolants & alcoylation \\
Oxydes acides & craquage \\
& polymérisation
\end{tabular}

Tableau IV. - Réactions et catalyseurs industriels les plus utilisés.

[Industrially important catalytic reactions and catalysts.]

Réactions Procédés

$\mathrm{H}_{2} \quad-\quad$ méthanation

réformage

Hydrogénation

traitement des huiles et lubrifiants

hydrogénation sélective d'oléfines

Déshydrogénation

désulfuration hydrogénante des pétroles

Oxydation

butane $\rightarrow$ butadiène

éthylbenzène $\rightarrow$ styrène

éthylène $\rightarrow$ acétate vinyle

$\mathrm{C}_{2} \mathrm{H}_{4} \rightarrow \mathrm{O}_{\mathrm{O}}^{\mathrm{CH}_{2}-\mathrm{CH}_{2}}$

$\mathrm{NH}_{3} \rightarrow \mathrm{HNO}_{3}$

Exemples

b : $\mathrm{Cu}, \mathrm{Ag}, \mathrm{Au}$

VIII : $\left\{\begin{array}{l}\mathrm{Fe}, \mathrm{Co}, \mathrm{Ni} \\ \mathrm{Ru}, \mathrm{Rh}, \mathrm{Pd} \\ \mathrm{Os}, \mathrm{Ir}, \mathrm{Pt}\end{array}\right\}$

$\mathrm{NiO}, \mathrm{Fe}_{2} \mathrm{O}_{3}, \mathrm{Fe}_{3} \mathrm{O}_{4}$

Co-Mo-O,

$\mathrm{ZnO}, \mathrm{Cr}_{2} \mathrm{O}_{3}$

Ni-Mo-O

$\mathrm{Sb}-\mathrm{Sn}-\mathrm{O}, \mathrm{Mo-Bi}-\mathrm{O}$ etc.

$\mathrm{MgO}, \mathrm{SiO}_{2}, \mathrm{Al}_{2} \mathrm{O}_{3}$

$\mathrm{SiO}_{2}-\mathrm{Al}_{2} \mathrm{O}_{3}$

zéolithes

$\mathrm{H}_{3} \mathrm{PO}_{4}, \mathrm{H}_{2} \mathrm{SO}_{4}$

$\mathrm{SO}_{2} \rightarrow \mathrm{H}_{2} \mathrm{SO}_{4}$

benzène ou n-butène ou n-butane $\rightarrow$ anhydride

maléique

o-xylène ou naphtalène $\rightarrow$ anhydride phtalique

$\mathrm{CH}_{3} \mathrm{OH} \rightarrow$ formol

$\mathrm{CO}$ et hydrocarbures (oxydation totale)

Ammoxydation

$\mathrm{CH}_{3}-\mathrm{CH}=\mathrm{CH}_{2} \rightarrow$ acrylonitrile

Catalyseurs

$\mathrm{Ni}, \mathrm{Fe}, \mathrm{Pt}-\mathrm{Re}$

supportés sur $\mathrm{SiO}_{2}, \mathrm{Al}_{2} \mathrm{O}_{3}$ ou zéolithes

Ni-Raney

$\mathrm{Ni}-\mathrm{Cr}$ ou Pd sur alumine

$\mathrm{Co}-\mathrm{Mo}-\mathrm{O}$

$\mathrm{Cr} / \mathrm{Al}_{2} \mathrm{O}_{3}$

oxyde de $\mathrm{Fe}$

Pd

$\mathrm{Ag} / \mathrm{Al}_{2} \mathrm{O}_{3}$

Pt-Rh

$\mathrm{V}_{2} \mathrm{O}_{5}$

$\mathrm{V}_{2} \mathrm{O}_{5}$

$\mathrm{V}_{2} \mathrm{O}_{5}$

Fe-Mo-O; Ag

$\mathrm{Pt} / \mathrm{Al}_{2} \mathrm{O}_{3}$

chlorure de vinyle

U-Sb-O

Oxy chloruration

Craquage

Isomérisation

hydrocarbures

Synthèse organique

Polymérisation

Friedel Craft

stéréo polymères

$\mathrm{CuCl}_{2}$ supporté

acides (silices-alumines zéolithes...)

$\mathrm{AlCl}_{3}$

alcoyles d'Al Ti et $\mathrm{V}, \mathrm{Cr}$ 
sur : les rendements, le type de matière première, la récupération ou la nécessité d'énergie, le prix de l'installation, le type et la quantité de sous-produits rejetés (problèmes de santé et écologiques). Le prix du catalyseur n'intervient souvent qu'au second rang !

Nous avons présenté dans les tableaux III et IV les principaux catalyseurs et des réactions typiques concernées ainsi que les catalyseurs les plus utilisés.

Ces catalyseurs peuvent être utilisés seuls ou déposés sur un support plus ou moins inerte tels $\mathrm{Al}_{2} \mathrm{O}_{3}, \mathrm{SiO}_{2}$ ou $\mathrm{MgO}$ essentiellement. L'utilisation d'un support peut permettre principalement d'accroître très sensiblement le rapport surface/volume de la phase active (en particulier pour les métaux précieux utilisés à moins de $1 \%$ pondéral) et parfois d'améliorer les propriétés catalytiques, par exemple dans le cas des zéolithes.

Dans le tableau V nous présentons quelques exemples sur les conditions réactionnelles pour certaines réactions d'oxydation ménagée. Ces exemples ont pour but de présenter au lecteur une idée concrète sur les conditions expérimentales en catalyse industrielle.

Nous allons considérer maintenant les techniques physiques les plus couramment utilisées en catalyse hétérogène pour la caractérisation des solides. Nous ne parlerons volontairement pas de techniques importantes mais d'applications limitées pour les catalyseurs réels telles la diffraction des électrons lents, la photoémission angulaire, la diffraction et diffusion des neutrons, les techniques de type Raman, Mole, etc...

Nous considérons :

1) les techniques qui, par principe, analysent l'ensemble du matériau et que nous désignerons comme techniques de volume même si nous portons un accent particulier aux caractérisations de phases adsorbées en surface;
2) les techniques qui analysent les toutes premières couches atomiques proches de la surface et que nous appellerons techniques de surface même si plusieurs couches sous la surface sont concernées.

3. Techniques physiques de volume - 3.1 MÉTHODES DE SPECTROSCOPIE MOLÉCULAIRE [1]. - Il s'agit essentiellement des techniques optiques d'infrarouge, uv-visible et Raman. Le tableau VI donne une idée des domaines d'énergie ou de longueurs d'ondes concernées et parallèlement des transitions mises en jeu.

Dans les spectroscopies UV et IR on enregistre la lumière absorbée (ou parfois émise) correspondant aux transitions d'un niveau énergétique à un autre. En spectroscopie Raman on analyse la lumière diffusée dont l'écart énergétique par rapport à l'énergie incidente, fournie généralement par un Laser, correspond à des différences entre niveaux énergétiques vibrationnels ou rotationnels. Les informations obtenues par ces trois techniques seront donc parfois analogues et parfois complémentaires puisque les domaines d'énergie sont différents donc les transitions concernées également.

On sait que l'énergie totale des niveaux orbitaux d'un composé peut être écrite :

$$
E_{0}=E_{\mathrm{T}}+R_{\mathrm{r}}+E_{\mathrm{v}}+E_{\mathrm{e}}
$$

représentant la sommation des énergies de translation, de rotation, de vibration et électronique avec $E_{\mathrm{e}} \gg E_{\mathrm{v}} \gg E_{\mathrm{R}}$. $E_{\mathrm{e}}$ intéresse le domaine UV, $E_{\mathrm{v}}$ le domaine IR et $E_{\mathrm{R}}$ le domaine micro-ondes. On voit donc que ces différentes spectroscopies permettront d'obtenir des informations complémentaires sur les états énergétiques d'un système donné.

Pour expliciter un peu ces notions nous pouvons

Tableau V. - Procédés industriels de formation de produits oxygénés en catalyse hétérogène.

[Industrial processes for the production of oxygenated products in heterogeneous catalysis.]

\begin{tabular}{|c|c|c|c|}
\hline $\begin{array}{c}\text { Elément de base } \\
\text { du catalyseur }\end{array}$ & Réaction & $\begin{array}{c}\text { Conditions } \\
\text { de température }\left({ }^{\circ} \mathrm{C}\right)\end{array}$ & $\begin{array}{c}\text { Rendement } \\
(\%) \\
\end{array}$ \\
\hline $\mathrm{MoO}_{3}$ & $\begin{array}{l}\mathrm{CH}_{3} \mathrm{OH} \rightarrow \text { formol } \mathrm{HCHO} \\
\text { propène } \rightarrow \text { acide acrylique ( } 2 \text { étapes) }\end{array}$ & $250-300$ & $\begin{array}{c}90-95 \\
85\end{array}$ \\
\hline $\mathrm{V}_{2} \mathrm{O}_{5}$ & $\begin{array}{l}\begin{array}{l}\text { benzène } \\
\text { n-butènes } \\
\text { n-butane } \\
\text { naphtalène } \\
\text { o-xylène }\end{array} \rightarrow \begin{array}{l}\text { anhydride } \\
\text { maléique }\end{array} \\
\begin{array}{l}\text { anhydride } \\
\text { phtalique }\end{array}\end{array}$ & $\begin{array}{l}400-450 \\
350-450 \\
350-550\end{array}$ & $\begin{array}{l}70-72 \\
45 \\
40-45 \\
81-85 \\
75-80\end{array}$ \\
\hline \multirow[t]{3}{*}{$\mathrm{Ag}$} & $\mathrm{C}_{2} \mathrm{H}_{4} \rightarrow \mathrm{C}_{2} \mathrm{H}_{4} \mathrm{O}$ & $\begin{array}{c}200-300 \\
(10-30 \mathrm{~atm} .)\end{array}$ & $70-75$ \\
\hline & $\mathrm{CH}_{3} \mathrm{OH} \rightarrow \mathrm{HCHO}$ & $450-600$ & $86-91$ \\
\hline & $\begin{array}{l}\text { alcool iso- } \\
\text { propylique }\end{array} \rightarrow$ acétone & $400-600$ & $85-90$ \\
\hline $\mathrm{Pd}$ & $\begin{array}{l}\mathrm{C}_{2} \mathrm{H}_{4}+\mathrm{CH}_{3} \mathrm{COOH} \stackrel{\mathrm{O}_{2}}{\rightarrow} \\
\text { acétate de vinyle }\end{array}$ & $\begin{array}{c}175-200 \\
(2-10 \text { atm. })\end{array}$ & $91-95$ \\
\hline
\end{tabular}


Tableau VI. - Comparaison des rayonnements électromagnétiques.

[Comparison of electromagnetic radiations.]

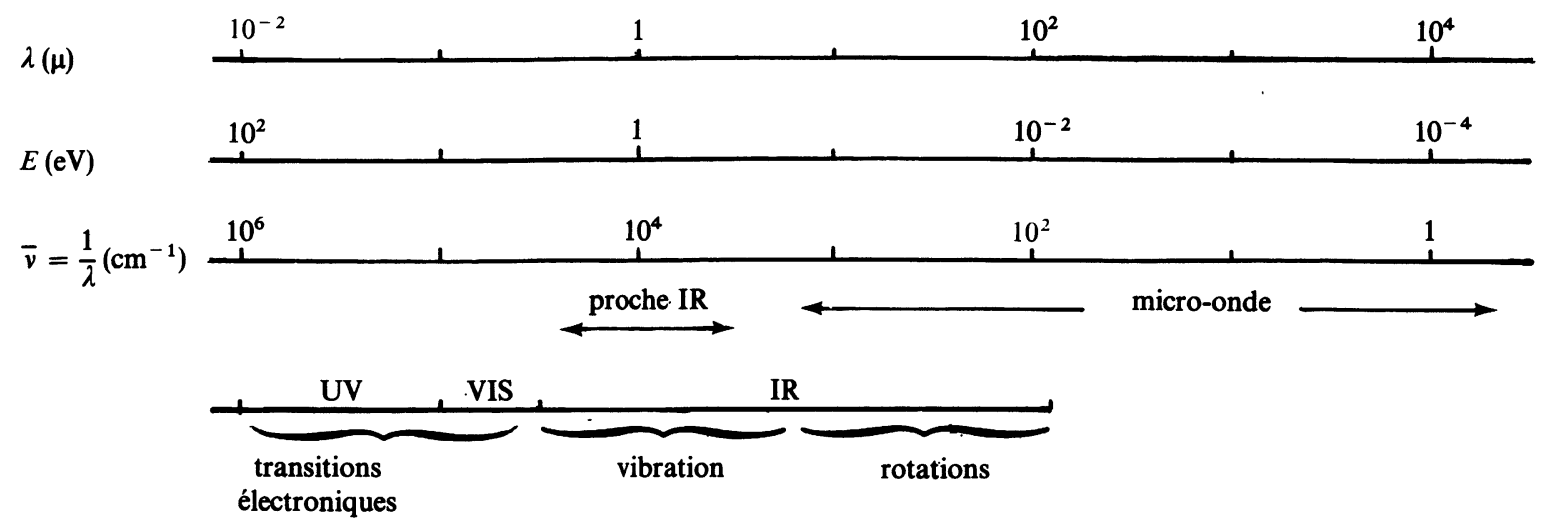

rappeler qu'un ion de transition présentant 5 niveaux énergétiques $\mathrm{d}$ dégénérés à l'état libre voit cette dégénérescence levée par le champ cristallin créé par l'environnement du cation selon le schéma présenté dans la figure 1. Cette levée de dégénérescence dépend de la symétrie environnante, des distorsions éventuelles et son amplitude dépend de la nature des ligandes et des longueurs de liaison. On conçoit alors que,

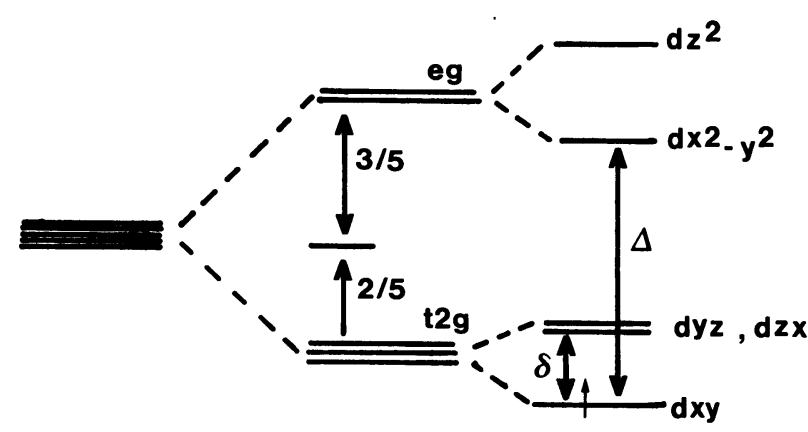

Oh

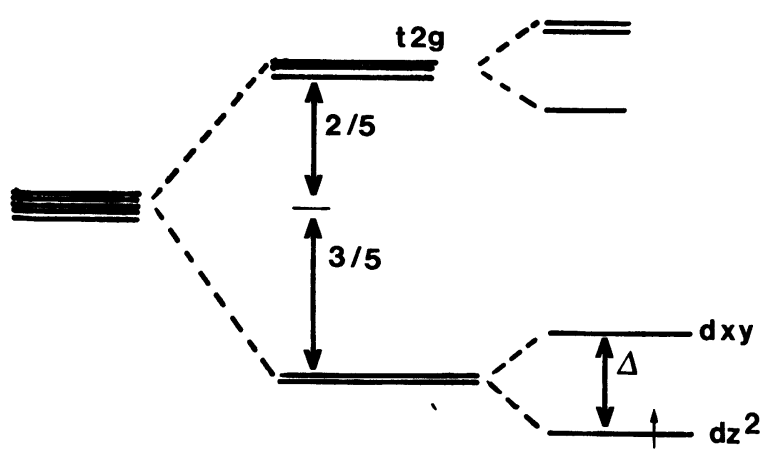

Td

\section{distortion}

Fig. 1. - Influence de la symétrie octaédrique $(\mathrm{Oh})$ ou tétraédrique (Td) et de distorsions tétragonales sur la levée de dégénérescence des niveaux d'un ion de transition.

[Influence of environmental octahedral or tetrahedral symmetries ( $\mathrm{Oh}$ or $\mathrm{Td}$ ) and tetragonal distortions on the degeneracy extent of d ions levels.] lorsque l'on pourra induire des transitions entre ces différents niveaux, on aura des enseignements importants sur la symétrie concernée, les distorsions éventuelles et les amplitudes du champ cristallin.

Dans le cadre de la Catalyse ces spectroscopies pourront être utilisées dans les conditions d'une réaction c'est-à-dire sous atmosphère contrôlée et à une température désirée. Notons cependant qu'on les utilise le plus souvent dans des conditions plus aisées d'étude (température ambiante, atmosphère de vide ou d'air) de façon à avoir des informations sur le catalyseur lui-même.

La spectroscopie IR apportera de précieux renseignements dans les domaines suivants :

a) Détermination des groupements fonctionnels organiques ou minéraux tels que ceux présentés ci-dessous (Tableau VII), groupements de surface (adsorbats) ou de volume (vibration de réseau).

Tableau VII. - Domaines de fréquences de vibration IR pour quelques groupements fonctionnels usuels. [IR vibrational frequences domains for some fonctionnal groups.]

\begin{tabular}{cc|cc} 
Groupements & $\begin{array}{c}\text { Fréquence IR } \\
\bar{v}\left(\mathrm{~cm}^{-1}\right)\end{array}$ & $\begin{array}{c}\text { Groupements } \\
\mathrm{C}-\mathrm{H}\end{array}$ & $\begin{array}{c}\text { Fréquence IR } \\
\bar{v}\left(\mathrm{~cm}^{-1}\right)\end{array}$ \\
- & - & - & - \\
$\mathrm{OH}$ & $3700-3300$ & aliphatique & $2990-2850$ \\
$\mathrm{NH}$ & $3500-3200$ \\
$\mathrm{CH}$ & $3300-2800$ & oléfinique & $3095-2950$ \\
$\mathrm{C}=\mathrm{C}$ & $1500-1690$ & aromatique & $3080-3030$ \\
$\mathrm{Si}-\mathrm{O}$ & $1100-700$ & acétylénique & $3400-3300$
\end{tabular}

b) Caractérisation des propriétés acido-basiques superficielles des catalyseurs solides. Pour cela on adsorbe sur le solide une base, comme l'ammoniac ou la pyridine, ou un acide et l'on analyse les spectres infra-rouge obtenus. La nature de l'entité obtenue, caractérisée par sa fréquence, permet de déterminer le site d'adsorption - par exemple un proton acide $\mathrm{H}^{+}$ (dit site Brönsted) ou un centre accepteur d'un doublet électronique (dit site de Lewis comme - Al). 
Tableau VIII. - Nombres d'onde IR $\left(\bar{v}\right.$ en $\left.\mathrm{cm}^{-1}\right)$ de la pyridine selon la nature du site d'adsorption.

[Wavenumber in $\mathrm{cm}^{-1}$ for pyridine adsorbed as a function of the nature of the adsorption site.]

Modes de vibration

$\begin{array}{lcccc} & 19 \mathrm{~b} & 19 \mathrm{a} & 8 \mathrm{~b} & 8 \mathrm{a} \\ \text { Pyridine libre } & \overline{-} & -\overline{4} & \overline{-} & \overline{-} \\ \text { Pyridine sur site de Lewis } & 1439 & 1478 & 1572 & 1583 \\ \text { Ion pyridinium } & 1440-1460 & 1470-1490 & 1562-1575 & 1610-1628 \\ & 1525-1540 & 1480-1490 & 1600-1613 & 1631-1640\end{array}$

On montre par exemple sur la figure 2 les variations d'acidité d'une zéolithe de type $\mathrm{Y}$ en fonction de la température d'activation du solide. Ces variations sont d'ailleurs comparables à celles des bandes d'absorption dues aux groupements $\mathrm{OH}$ (sites de Brönsted) qui disparaissent par calcination avec formation de sites de Lewis. Notons que les nombres d'onde augmentent avec la force acide ce qui permet d'avoir une idée de l'acidité comparative entre échantillons (Tableau VIII).

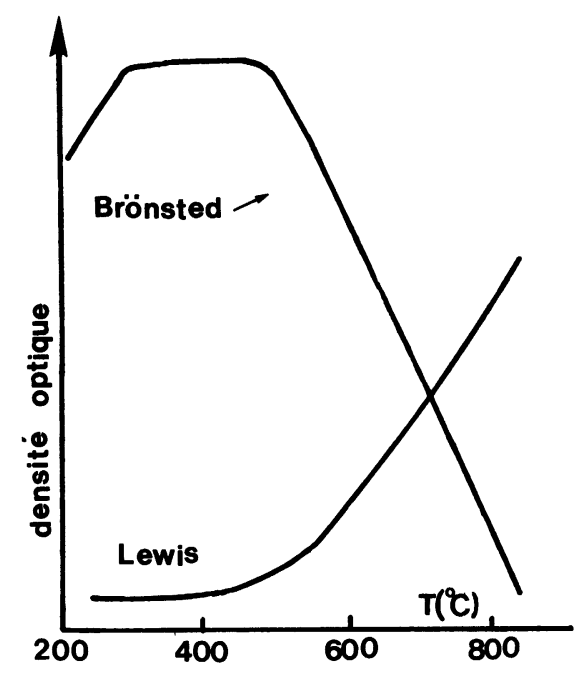

Fig. 2. - Evolution de la concentration en sites protoniques et en centres de Lewis en fonction de la température d'activation préalable, déduite de l'intensité des bandes IR à 1540 et $1450 \mathrm{~cm}^{-1}$ respectivement après adsorption de pyridine (d'après [2]) sur zéolithe $\mathrm{Y}$.

[Variations of optical density of IR bands at 1540 (pyridinium ion) and $1450 \mathrm{~cm}^{-1}$ (Lewis adsorbed pyridine) as a function of activation temperature of $\mathrm{Y}$ zeolite samples prior to pyridine adsorption (from [2]).]

c) Caractérisation des complexes : La spectroscopie IR étant sensible aux groupements fonctionnels, on conçoit aisément que lorsqu'une réaction catalytique fait intervenir des complexes de coordination qui évoluent dans le temps, des informations importantes pourront être obtenues. Prenons, comme exemple, la réaction de carbonylation du méthanol $\left(\mathrm{CH}_{3} \mathrm{OH}+\mathrm{CO} \rightarrow \mathrm{CH}_{3} \mathrm{COOH}\right)$ catalysée par les ions $\mathrm{Rh}$ en présence d'iodure de méthyle [3]. Nous avons représenté sur la figure 3 les différences des bandes d'adsorption IR en fonction du complexe carbonyl correspondant.

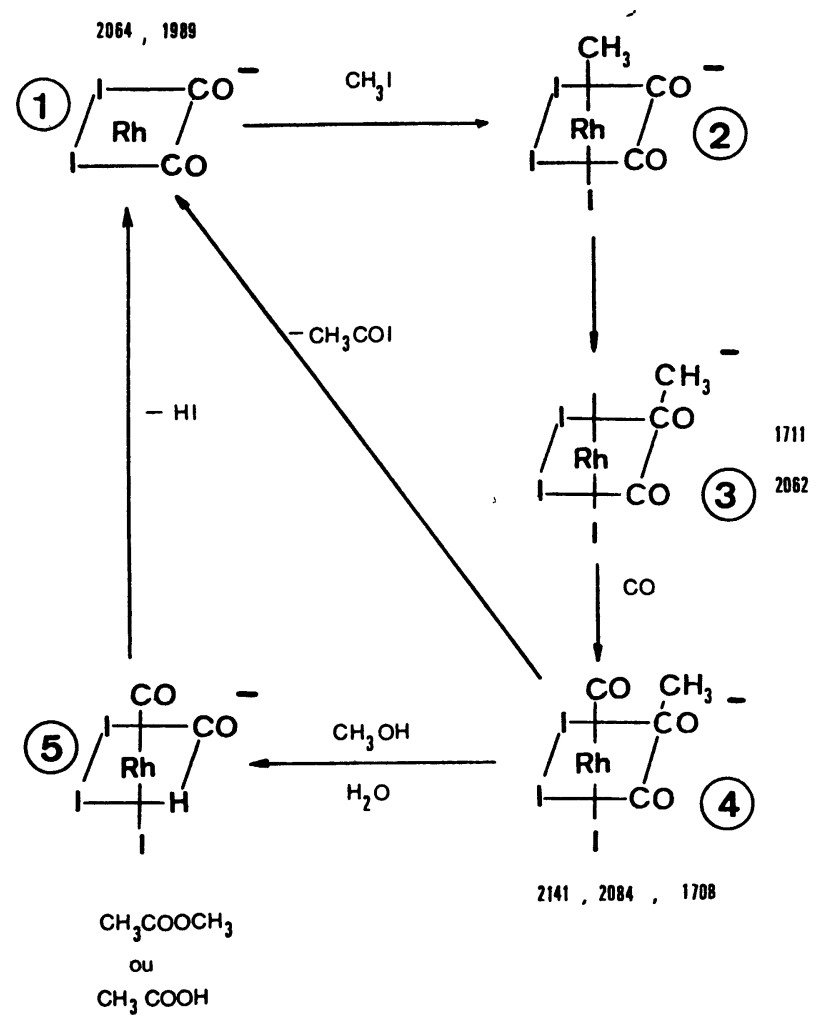

Fig. 3. - Mécanisme proposé pour la réaction de carbonylation du méthanol sur catalyseur au $\mathrm{Rh}$ en présence de $\mathrm{CH}_{3} \mathrm{I}$. Les fréquences IR notées correspondent aux groupements carbonyles des différents complexes des stades intermédiaires (cf. [3]).

[Mechanism proposed in ref. [3] for the reaction of carbonylation of methanol on Rh catalyst in presence of $\mathrm{CH}_{3} \mathrm{I}$. The IR frequencies as indicated are refering to carbonyl groups for differents complexes of intermediate steps.]

d) Caractérisation d'une surface à l'aide de sondes appropriées. On utilise fréquemment $\mathrm{CO}$ qui donne des bandes IR assez étroites et permet de caractériser les propriétés superficielles - par exemple donneur ou accepteur d'électron - d'un solide. Des exemples sont donnés dans les communications de MM. Martin et Mathieu [20]. La spectroscopie UV [4] fait intervenir des transitions électroniques qui peuvent être classées en plusieurs groupes selon la nature des sauts électroniques :

a) Transitions d-d dans le cas des ions de transition sont caractéristiques par leur position énergétique du degré d'oxydation de l'ion et de la symétrie environnante ( $\mathrm{Oh}, \mathrm{Td}$, distorsions). 
b) Transferts de charge : impliquent un transfert d'électron d'une orbitale occupée vers une orbitale vide. Les bandes d'absorption obtenues sont caractéristiques du degré d'oxydation et des liaisons avec les ligandes.

c) Transitions $\pi-\pi^{*}$ et $n-\pi^{*}$, données essentiellement pour les carbocations ou radicaux insaturés, caractérisent des groupements fonctionnels particuliers.

Les informations que l'on obtiendra en spectroscopie UV-visible porteront sur :

a) Caractérisation des degrés d'oxydation des ions : cet aspect est important car il y a très peu de techniques permettant une telle détermination et constitue l'un des apports les plus importants de la technique.

$\beta$ ) Détermination de la symétrie environnante de l'élément analysé : cet aspect est important pour caractériser les complexes intervenant dans la réaction catalytique mais il est bon de garder à l'esprit que, principalement pour les solides, les bandes d'adsorption sont larges ce qui gêne considérablement les études fines, en particulier lorsqu'il y a recouvrement de bandes.

y) Caractérisation de groupements fonctionnels et par là des phases adsorbées. On pourra accéder à la caractérisation de complexes intermédiaires dans une réaction catalytique (radicaux allyles par exemple) ou des propriétés acido-basiques d'une surface solide.

La technique pourra être utilisée avec profit dans les conditions de la réaction catalytique à condition évidemment que l'absorption UV soit détectable.

\subsection{SPECTROSCOPIES MAGNÉTIQUeS (RPE ET RMN)} [5]. - Ce sont les spectroscopies qui font intervenir les moments magnétiques de spin (électronique et nucléaire). Ces spins sont séparés en deux familles (parallèle ou anti-parallèle) par un champ magnétique continu appliqué. Les spectres enregistrés correspondent à l'absorption d'énergie (micro-onde en RPE et radio fréquence en $\mathrm{RMN}$ ) nécessaire à la transition électronique ou nucléaire d'un niveau inférieur au niveau supérieur. Chaque niveau peut être déplacé ou subdivisé par des interactions avec l'environnement de l'élément considéré. Ce sera là toute la richesse de ces techniques.

En spectroscopie de résonance paramagnétique électronique on ne pourra analyser que les composés ayant un certain nombre - en général un - d'électrons non appariés, c'est-à-dire seulement les composés paramagnétiques. Dans le cas de la présence de plusieurs degrés d'oxydation d'un élément on ne détectera donc qu'une partie de ceux-ci : ceux qui sont paramagnétiques.

Un autre aspect important est la caractérisation de la symétrie environnante dans le cas de complexes d'ions paramagnétiques. Cette caractérisation, provenant de la détermination de ce que l'on appelle le facteur $g$, est due au couplage de l'électron dans son niveau fondamental avec différents niveaux excités vides (cf. Fig. 1). Un bon accord avec les données de spectroscopie UV, faisant intervenir les mêmes niveaux énergétiques, pourra alors être obtenu.

La spectroscopie de RPE sera également très utilisée pour la caractérisation des radicaux libres ou ions radicalaires. Par extension de cette application, on peut concevoir que, si une surface présente des sites accepteurs ou donneurs d'électron (oxydo-réducteur), par addition de composés à faible potentiel d'ionisation comme le pérylène ou l'anthracène ou à affinité électronique suffisante comme le tétracyanoéthylène ou le trinitrobenzène, il $\mathrm{y}$ a formation de cations ou anions radicalaires, respectivement. L'étude de ces ions radicalaires par RPE permettra d'avoir des informations importantes sur les propriétés acidobasiques ou oxydo-réductrices des catalyseurs solides.

La spectroscopie de RPE sera enfin utilisée pour l'étude des phases adsorbées, lorsque, bien sûr, ces phases sont paramagnétiques comme par exemple $\mathrm{O}^{-}, \mathrm{O}_{2}^{-}, \mathrm{O}_{3}^{-}, \mathrm{CO}_{2}^{-}, \mathrm{SO}_{2}^{-}$, etc. ou des complexes de type $\mathrm{Cu}\left(\mathrm{NH}_{3}\right)_{4}^{2+}$ par exemple. Cet aspect est important car on préconise souvent la présence de telles espèces au cours de la réaction catalytique à partir de l'étude des mécanismes réactionnels faisant intervenir par exemple des formes mono ou diatomiques de l'hydrogène ou de l'oxygène, des radicaux libres, des carbocations etc... L'observation de telles entités par RPE est donc importante mais leur non-observation ne signifie pas forcément qu'elles n'existent pas puisqu'il peut être question de durée de vie. Notons cependant que si on opère en continu et si la concentration instantanée est suffisante on pourrait observer de telles espèces fugaces puisque l'échelle de temps en RPE est de l'ordre de $10^{-10}$ seconde.

Rappelons encore que la sensibilité de la technique est extraordinaire puisqu'elle peut descendre au $100^{\mathrm{e}}$ de $\mathrm{ppm}$. La technique peut être utilisée dans des conditions proches de la réaction catalytique mais il y a de fortes limitations dues à des aspects secondaires importants comme la relaxation spin-réseau et la loi de Curie.

La caractérisation des complexes organométalliques peut être très fructueuse en RPE car si les ligandes possèdent des noyaux de spin nucléaire non nul la détermination du nombre, de l'intensité et de la séparation des raies hyperfines observées permet de remonter à la symétrie du complexe, au nombre et à la nature des ligandes.

La spectroscopie de RMN est utilisée également dans de nombreux domaines. On a déjà mentionné son utilisation pour l'analyse des produits de réaction, en particulier lorsque l'on utilise des molécules marquées. Les principales données de RMN sont :

- La caractérisation des groupements fonctionnels de Chimie $\mathrm{CH}, \mathrm{CH}_{2}, \mathrm{CH}_{3}, \mathrm{OH}, \mathrm{NH}$, etc.

- L'étude des phases adsorbées et espèces intermédiaires. Par exemple la formation d'espèces allyliques pourra être observée puisque les groupements $\mathrm{CH}$ sont distincts de ceux du réactif initial. La struc- 
ture électronique pourra également être définie à partir des déplacements chimiques observés.

- L'étude de la structure de complexes organiques, par exemple des complexes carbonyles d'ions de transition. Comme dans le cas des spectres IR (cf. Fig. 3) l'étude du déplacement chimique permettra de caractériser le complexe étudié et les éventuels transferts électroniques ion central-ligandes.

La technique de magnétisme [6], qui consiste à mesurer l'aimantation et la susceptibilité magnétique des composés, est d'une grande importance pour l'étude des composés magnétiques (ferro- ; anti-ferro-, para-) comme essentiellement $\mathrm{Fe}, \mathrm{Ni}, \mathrm{Co}$. On utilise avec succès la technique pour :

a) Caractériser la taille moyenne et la distribution de tailles de particules métalliques (granulométrie) comme G. A. Martin l'a présenté dans sa communication [20].

b) Déterminer le nombre de liaisons d'un adsorbat avec un substrat. Pour cela on détermine la baisse d'aimantation à saturation due à l'élimination de l'aimantation des atomes du catalyseur intervenant dans l'adsorption.

c) Définir le type de coordination. Ainsi par exemple l'aimantation à saturation et donc la susceptibilité magnétique de $\mathrm{Ni}^{2+}$ est égale par ion à 3,2 ou $4,1 \mu_{\mathrm{B}}$ pour une coordination octaédrique ou tétraédrique respectivement contre $2,8 \mu_{\mathrm{B}}$ pour l'ion libre.

d) Détermination du moment magnétique des ions, par exemple $\mathrm{Cr}^{3+}, \mathrm{Ni}^{2+}, \mathrm{Fe}^{3+}, \mathrm{Mo}^{5+}$, etc.

3.3 Spectroscopie Mössbauer (RGN) [7]. L'apport de cette technique est traité de façon beaucoup plus complète dans la communication de Bussière [20]. Citons simplement les principales informations que l'on peut obtenir dans le domaine de la catalyse.

a) Détermination $d u$ degré d'oxydation de l'ion considéré. Le cas du fer $\left(\mathrm{Fe}^{0}, \mathrm{Fe}^{2+}, \mathrm{Fe}^{3+}\right)$ est certainement le plus spectaculaire.

b) Mise en évidence de plusieurs phases distinctes comme dans le cas des ferrites. Evidemment la spectroscopie Mössbauer ne permet pas de caractériser sans ambiguité les différentes phases éventuelles mais elle permet de spécifier la présence de plusieurs phases par l'observation de plusieurs spectres. Par comparaison aux spectres purs obtenus pour des phases connues et éventuellement à l'aide de résultats d'autres techniques - en particulier la diffraction des rayons $\mathrm{X}$ - la détermination des différentes phases est possible.

c) Caractérisation de la symétrie environnante de l'élément étudié. En effet, la mesure du gradient de champ électrique par les interactions quadrupolaires permet de remonter au schéma énergétique de décomposition spectrale (cf. Fig. 1) et par là au rôle des coordinats, du degré de covalence, etc...

Notons que des éléments tels $\mathrm{Fe}, \mathrm{Sn}$ et $\mathrm{Sb}$, très communs pour les mesures Mössbauer, sont des éléments importants de nombreux catalyseurs de Fisher-Tropsch ( $\mathrm{CO}+\mathrm{H}_{2} \rightarrow$ hydrocarbures légers), de méthanation et d'oxydation ménagée. Enfin la technique peut être utilisée dans des conditions proches de la réaction catalytique.

3.4 LA MICROSCOPIE ÉleCtroniQue [8, 9]. - Cette technique est très répandue et son principal intérêt provient de son haut pouvoir de résolution qui peut atteindre 1,5 à $3 \AA$ et qui est dû à la faible longueur d'onde associée à l'électron. Cette longueur d'onde, pour une tension accélératrice de $100 \mathrm{kV}$, est $10^{5}$ fois plus petite que pour une radiation lumineuse. La technique est utilisée pour la détermination de la taille et de la forme des cristallites de matériaux solides allant jusqu'à celle de la texture et de la porosité. Dans le cas des catalyseurs métalliques supportés on peut effectuer une granulométrie comme le décrit G. A. Martin dans sa communication [20]. La principale difficulté réside dans une statistique suffisante dans la détermination de cette granulométrie c'est-àdire dans la représentativité de la parcelle d'échantillon analysé. La microscopie à balayage, dont le pouvoir de résolution est inférieur, permet d'avoir une bonne représentativité de la granulométrie puisqu'on analyse des plages d'échantillon de dimensions bien supérieures.

Le faisceau d'électrons peut aussi être utilisé en micro-diffraction et l'analyse des clichés obtenus permet d'avoir des informations importantes sur l'état cristallin, l'orientation de faces cristallines particulières et la nature des particules étudiées. On entrevoit immédiatement l'intérêt de la technique quand on se souvient que l'orientation de particules métalliques ou d'oxydes intervient dans les propriétés catalytiques et que l'hétérogénéité du solide est souvent élevée !

Des techniques fines de préparation telles les coupes, les répliques, les répliques extractives ou d'analyse tel le champ sombre ou l'analyse des électrons secondaires émis permettent encore d'améliorer les performances de la technique en isolant support et phase active ou en analysant l'intérieur des grains de catalyseurs.

Un accessoire important de la technique peut apporter également des informations précieuses à partir de l'analyse en énergie des rayons X émis sous l'impact électronique. Ce rayonnement $\mathrm{X}$ est caractéristique d'un élément donné et l'on a ainsi une carte de répartition de l'élément dans la particule étudiée : c'est la technique de micro-sonde [9]. On voit apparaître actuellement une génération de microscopes spécialement étudiés pour travailler en balayage et équipés de canons à émission de champ permettant d'obtenir des sondes électroniques de quelques $\AA$ de diamètre. On a ainsi la possibilité d'analyser des zones qu'une vingtaine d'Å de diamètre par émission $\mathrm{X}$ ou pertes d'énergie des électrons, donc d'avoir une identification 
des éléments. L'intérêt de cette technique pour l'étude des alliages métalliques et de l'hétérogénéité des catalyseurs du point de vue arrangements atomiques est de toute première importance pour l'avenir. Le seul handicap en est le prix très élevé de 3 à 4 millions de francs ! !

Notons enfin que la technique de microscopie nécessitant d'opérer sous vide n'est pas adaptée aux études dans les conditions de réaction catalytique. Une chambre de réaction au niveau de l'échantillon peut néanmoins être adaptée dans certains cas et permet de se rapprocher des conditions de travail réelles du catalyseur.

3.5 Diffraction Des Rayons X [10]. - Cette technique basée sur les applications de la loi de Bragg est bien connue. On détermine les diamètres des anneaux de diffraction et les variations d'intensités relatives des raies. On obtient ainsi des données sur :

a) Les variations des paramètres de réseau, par exemple lors de la formation d'alliage ou de solutions solides (loi de Végard).

b) La présence de plusieurs phases distinctes, lorsque leurs concentrations respectives sont suffisantes (> 5-10\%).

c) $\mathrm{La}$ reconstruction ou la modification éventuelle d'un réseau en cours de réaction.

d) La distribution des tailles des particules de solide à partir de l'élargissement des raies de diffraction (cf. communication de G. A. Martin à cette même réunion du GFSM [20]).

$e$ ) La présence de phases cristallines présentant éventuellement des orientations cristallographiques préférentielles.

Notons que l'on peut opérer dans des conditions proches de la réaction catalytique grâce à des chambres à atmosphère gazeuse et températures $\left(<800^{\circ} \mathrm{C}\right)$ contrôlées.

Une technique dérivée est basée sur la structure fine apparaissant au-delà du seuil d'excitation $\mathrm{X}$ et qui, à partir d'un traitement mathématique complexe à base de transformée de Fourier, permet de déduire les distances régulières atome à atome même si le solide n'est pas cristallisé. Il s'agit de l'EXAFS [11] (extended $\mathrm{X}$ ray absorption fine structure) qui nécessite des sources puissantes de rayons $X$ en énergie et en nombre de photons, fournis par de grands accélérateurs comme dans l'anneau de stockage DCI au laboratoire de LURE (Orsay) et par de puissants générateurs $\mathrm{X}$. Cette technique commence à être appliquée avec succès à des catalyseurs réels.

3.6 Conductivité ÉlectriQue [12]. - Cette technique consiste à déterminer la conductivité électrique des catalyseurs solides et à étudier les variations de cette conductivité en fonction de l'insertion d'un élément étranger dans un solide (solution solide). On peut ainsi déterminer si un solide est conducteur, semi-conducteur $\mathrm{n}$ ou $\mathrm{p}$ ou isolant. Certaines réactions catalytiques font en effet intervenir des processus d'oxydo-réduction et des transferts électroniques dans le solide. On conçoit ainsi que les modifications de conductivité interviendront sur les propriétés catalytiques des solides, en particulier pour les oxydes et dans les réactions d'oxydation ménagée. Cette technique est difficilement utilisable dans les conditions de réaction et est soumise à une certaine incertitude du fait des problèmes de contact électrique aux joints de grain pour catalyseurs pulvérulents.

4. Méthodes de surface. - Il n'est pas question dans cette revue de détailler toutes les techniques de surface et nous nous limiterons aux techniques d'ESCA et de SIMS et ISS dont les applications en catalyse sont importantes.

Comme pour les autres spectroscopies on envoie sur un matériau un faisceau incident et on analyse en nature et énergie le faisceau émergent. Les techniques de surfaces correspondent aux techniques pour lesquelles le faisceau incident et/ou émis sera composé de particules de masse non négligeables comme les électrons, les neutrons ou les ions qui seront absorbés très facilement par la matière et n'analyseront donc que les toutes premières couches atomiques superficielles.

D'un autre côté et pour la même raison d'absorption, ces techniques nécessiteront d'opérer sous un vide poussé $\left(10^{-8}-10^{-10}\right.$ torr) c'est-à-dire dans des conditions très éloignées de celles de réactions catalytiques et seront particulièrement fructueuses pour les solides à cause des faibles tensions de vapeur. Néanmoins, la connaissance de la surface d'un catalyseur par rapport à son volume est du tout premier intérêt en catalyse et de gros efforts sont actuellement portés sur ce domaine.

\subsection{SPECTROSCOPIE DE PHOTO ÉLECTRONS : ESCA OU} XPS [13]. - Le principe de cette technique est assez simple : un faisceau de rayons $\mathrm{X}$

$$
(\mathrm{AlK} \alpha: 1486,6 \mathrm{eV} \text { ou } \mathrm{MgK} \alpha: 1253,6 \mathrm{eV})
$$

frappe l'échantillon et il y a émission d'électrons qui sont analysés en nombre et en énergie. L'énergie cinétique $E_{\mathrm{c}}$ des électrons émis est reliée à l'énergie de liaison $E_{1}$ des électrons dans leurs niveaux orbitaux correspondants et à l'énergie $h v$ des photons incidents par la relation :

$$
h v=E_{\mathrm{c}}+E_{1}+\varphi
$$

où $\varphi$ est la fonction d'extraction du spectromètre.

La détermination précise de cette énergie de liaison permet d'obtenir deux informations importantes :

a) La nature de l'élément concerné. Tous les éléments constitutifs d'un matériau pourront être identifiés(sauf l'hydrogène) grâce à la détermination des énergies de liaison caractéristiques des éléments. Cette pro- 
priété est très importante et est à l'origine du nom ESCA : Electron spectroscopy for chemical analysis.

b) Le degré d'oxydation de l'élément analysé car la valeur de l'énergie de liaison mesurée croît en première approximation avec la charge positive d'un ion qui dépend du degré d'oxydation et du degré de covalence. L'analyse détaillée se fera par comparaison avec des échantillons connus contenant l'élément à analyser à un degré d'oxydation connu.

Une analyse des surfaces relatives des différents pics associée à la section efficace pour l'orbitale électronique concernée (valeurs connues dans des tables) et au libre parcours moyen des électrons, fonction de leur énergie cinétique (en racine carrée en première approximation) permet d'obtenir des données quantitatives relatives, c'est-à-dire des données sur les proportions relatives des différents éléments constitutifs d'un matériau.

On conçoit alors toute la puissance de la technique qui permettra de distinguer à la surface $(\sim 20$ à $50 \AA$ de profondeur) des solides les proportions des différents éléments et leur degré d'oxydation. On pourra ainsi détecter des ségrégations éventuelles et des modifications que pourrait amener la réaction catalytique. Nous verrons dans les exemples choisis $(\S 6)$ comment de telles propriétés peuvent être mises en valeur dans la connaissance des catalyseurs.

Notons que la surface analysée est encore de l'ordre $\mathrm{du} \mathrm{cm}^{2}$ et qu'un développement vers les techniques de balayage serait très fructueux.

4.2 SECONDARY ION MASS SPECTROSCOPY : SIMS [14] ET ION SCATTERING SPECTROSCOPY : ISS [4]. - Dans cette technique un faisceau d'ions argon est envoyé sur une surface et on analyse la nature des ions émis par spectrométrie de masse, c'est le SIMS, ou les variations d'énergie des ions argon que l'on relie aux ions de surfaces, c'est l'ISS. Cette technique est vraiment de surface puisque l'on peut vraiment analyser la première couche atomique et les ensembles d'atomes analysés sont représentatifs des arrangements de surface. La technique est cependant très limitée et d'interprétation soumise à un certain doute dans la mesure où la perturbation de la surface est importante et les recombinaisons et réarrangements des ions sont possibles avant la détection. De plus, la détermination des arrangements initiaux est très délicate et nécessite souvent l'analyse d'échantillons témoins connus. De plus le décapage ionique peut être sélectif.

Néanmoins cette technique permet d'avoir des enseignements sur les arrangements atomiques de la surface et à notre connaissance et malgré ses défauts, elle demeure la seule possibilité pour une telle analyse dont nous avons montré l'importance en catalyse.

5. Analyse critique. - La présentation ci-dessus des principales propriétés des méthodes physiques appliquées à la caractérisation des catalyseurs industriels réels n'est volontairement pas exhaustive. Elle ne concerne que la partie qui implique les applications à la caractérisation des catalyseurs et des phases adsorbées. Une analyse des propriétés des différentes méthodes et de leurs applications montre d'une part que l'emploi de plusieurs techniques est souvent nécessaire (cf. § 6) si l'on veut obtenir des informations à la fois sur les caractéristiques massiques et de surface d'un solide et d'autre part que l'étude de tel ou tel problème nécessite des techniques appropriées. En d'autres termes l'application d'une technique dépend de l'information recherchée et également du catalyseur étudié. Ainsi pour des composés absorbant la lumière (noirs en particulier) les spectroscopies UV et infrarouge ne pourront pas être utilisées. Pour des métaux ou des conducteurs électriques il faudra proscrire la RPE. Pour des composés ayant des impuretés magnétiques (le fer en particulier) les techniques de magnétisme et même de RMN seront à considérer avec précaution. Pour les composés instables les techniques sous vide (ESCA, microscopie électronique...) sont fortement déconseillées.

Comme aperçu général on peut dire que très peu de techniques permettent de caractériser le matériau dans les conditions mêmes de la réaction catalytique. Citons les spectroscopies UV-vis., IR, Mössbauer et de diffraction des rayons $\mathrm{X}$ avec les limitations citées plus haut. Il n'en demeure pas moins que les informations obtenues hors des conditions de réaction permettent de caractériser le catalyseur et d'approcher les sites catalytiquement réactifs, en particulier les arrangements atomiques de surface. Il faut également noter que les caractéristiques massiques jouent également un rôle que l'on pourrait schématiser comme celui d'un support de phase active avec comme effet la dispersion de la phase active ou la conductivité électrique pour assurer les transferts d'électrons nécessaires à la réaction. Si chaque technique apparaît comme apportant ses propres informations très spécifiques, les techniques d'applications les plus larges et les plus importantes actuellement me paraissent être les microscopies électronique et analytique et les techniques de surface EXAFS de surface, XPS, UPS et ISS. Ceci ne signifie nullement que les autres techniques n'ont aucun intérêt mais il m'apparaît que l'avancement futur de nos connaissances sur les sites catalytiques passera principalement par ces techniques capables de préciser les arrangements atomiques de surface.

\section{Exemples de caractérisation des catalyseurs. -}

Nous avons choisi trois seuls exemples pour permettre au lecteur de se faire une idée sur l'importance de l'emploi simultané de plusieurs techniques physiques. D'autres exemples ont été présentés dans les communications de P. Bussière (Mössbauer), G. A. Martin (rayons $\mathrm{X}$, microscopie électronique, magnétisme, titration chimique $\mathrm{H}_{2} / \mathrm{O}_{2}$ ) et $\mathrm{M}$. V. Mathieu (spectroscopies optiques) [20]. 
6.1 INSERTION D'UN ÉLÉMENT ÉTRANGER DANS L'OXYDE DE TITANE [15]. - Pour cette opération on imprègne une poudre de $\mathrm{TiO}_{2}$ avec un sel de molybdène et on calcine l'ensemble à $500^{\circ} \mathrm{C}$ sous air. En partant de paramolybdate d'ammonium et à l'aide de la spectroscopie UV on observe la décomposition du molybdate $\left(\mathrm{Mo}^{6+}\right)$ au-dessus de $100{ }^{\circ} \mathrm{C}$, l'insertion $\mathrm{du}$ molybdène sous forme de $\mathrm{Mo}^{5+}$ en symétrie tétraédrique déformée dans le réseau de l'anatase à $500^{\circ} \mathrm{C}$ et sous forme de $\mathrm{Mo}^{5+}$ selon deux arrangements distincts tétraédriques dans le réseau du rutile en calcinant à plus haute température $\left(800^{\circ} \mathrm{C}\right)(\mathrm{Fig}$. 4)

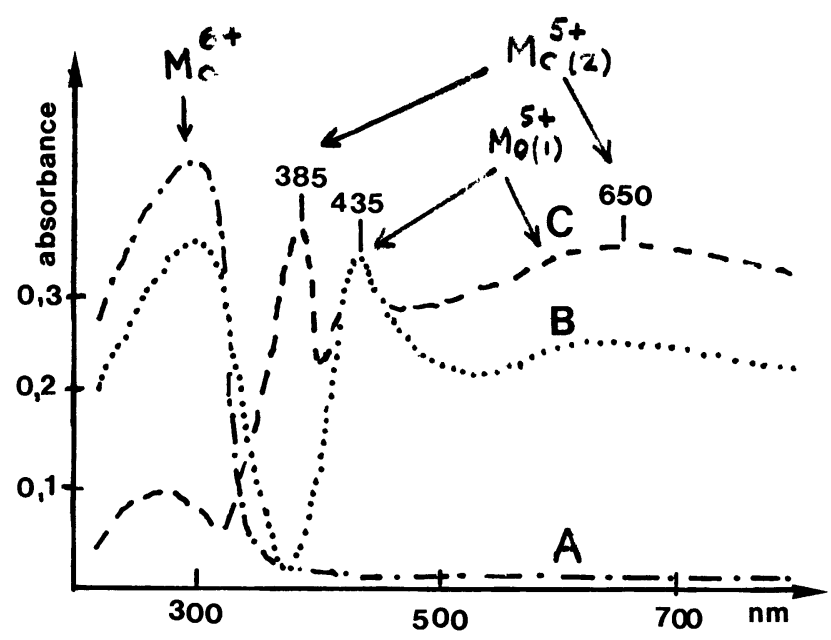

Fig. 4. - Spectre UV-visible d'un échantillon à $0,51 \%$ atomique Mo dans $\mathrm{TiO}_{2}$ préparé par coprécipitation (A) séché à l'air à $110^{\circ} \mathrm{C}$, (B) calciné sous air à $500^{\circ} \mathrm{C}$ (anatase) (C) calciné sous air à $800^{\circ} \mathrm{C}$ (rutile).

[UV spectra of 0.51 atom percent of $\mathrm{Mo}$ in $\mathrm{TiO}_{2}$ sample prepared by coprecipitation and after : (A) drying at $110^{\circ} \mathrm{C}$ in air, (B) calcination in air at $500^{\circ} \mathrm{C}$ (anatase) and (C) calcination at $800^{\circ} \mathrm{C}$ in air (rutile).]

Une étude par RPE permet de détecter $\mathrm{Mo}^{5+}$ (ion $\mathrm{d}^{1}$ ) en position réticulaire (la teneur en Mo est inférieure à $1 \%$ ) et montre que le réseau du rutile présente une meilleure cristallisation que celui de l'anatase. Cet effet de la température sur l'état de cristallisation des oxydes et l'insertion d'un ion étranger est bien connu.
On arrive ainsi à la conclusion que les échantillons précédents calcinés à 500 ou $800^{\circ} \mathrm{C}$ correspondent à $\mathrm{Mo}^{5+}$ inséré dans $\mathrm{TiO}_{2}$.

Nous avons effectué parallèlement une étude par ESCA sur ces deux échantillons et observé les propriétés étonnantes résumées dans le tableau IX.

On constate que l'échantillon calciné à $500^{\circ} \mathrm{C}$ contient environ $2 / 3 \mathrm{Mo}^{6+}$ et $1 / 3 \mathrm{Mo}^{5+}$ et que la mesure quantitative à partir de l'intensité des pics ESCA est en bon accord avec l'analyse chimique. Ces résultats sont tout à fait cohérents avec ceux obtenus à partir de la spectroscopie UV.

Par contre si l'on considère l'échantillon calciné à $800^{\circ} \mathrm{C}$ on constate d'une part que la concentration relative $\mathrm{Mo}: \mathrm{Ti}$ a augmenté d'un facteur 23 alors que l'analyse chimique ne décèle aucune différence et d'autre part que le molybdène est essentiellement au degré d'oxydation +6 . Ces résultats sont tout à fait opposés à ceux de spectroscopie UV qui n'indiquent que la présence de $\mathrm{Mo}^{5+}$ en majorité.

En fait il apparaît, puisque la technique d'ESCA permet d'analyser la surface des grains de catalyseur alors que les spectroscopies UV et RPE analysent l'ensemble, que l'on a affaire à une ségrégation de Mo vers la surface des grains sous l'effet thermique parallèlement à l'insertion de $\mathrm{Mo}^{6+}$ dans le réseau à l'état $\mathrm{Mo}^{5+}$.

Finalement le solide calciné à $800^{\circ} \mathrm{C}$ correspond vraisemblablement à l'oxyde $\mathrm{MoO}_{3}$ dispersé à la surface du rutile dopé par $\mathrm{Mo}^{5+}$.

Notons que l'ESCA permet de mettre en évidence cette ségrégation sans ambiguïté. Cependant l'arrangement superficiel réel en surface, en particulier s'il s'agit bien de $\mathrm{MoO}_{3}$, reste inconnu ou spéculatif.

\subsection{SOlution SOLIDE ET SÉGRÉGation DE PHASE. -} Un phénomène analogue a été observé dans le cas des systèmes $\mathrm{Sb}-\mathrm{Sn}-\mathrm{O}[16]$. De tels catalyseurs sont préparés généralement par coprécipitation à $\mathrm{pH} \simeq 7$ d'une solution en proportions choisies et variables en sels des deux cations, la teneur en $\mathrm{Sb}$ par rapport à $\mathrm{Sn}$ pouvant varier de 0 à $100 \%$. Des mesures de conductivité électrique [17] de Mössbauer (de Sb) [18]

Tableau IX. - Résultats d'ESCA obtenus pour un échantillon $\mathrm{Mo} / \mathrm{TiO}_{2}$ de teneur atomique de $0,5 \%$ en Mo calciné à 500 ou $800^{\circ} \mathrm{C}$. Les énergies de liaison sont en $\mathrm{eV}( \pm 0,2)$.

[ESCA results on $0,5 \% \mathrm{Mo} / \mathrm{TiO}_{2}$ catalyst calcined at 500 or $800{ }^{\circ} \mathrm{C}$. Binding energies in $\mathrm{eV}( \pm 0.2 \mathrm{eV})$.]

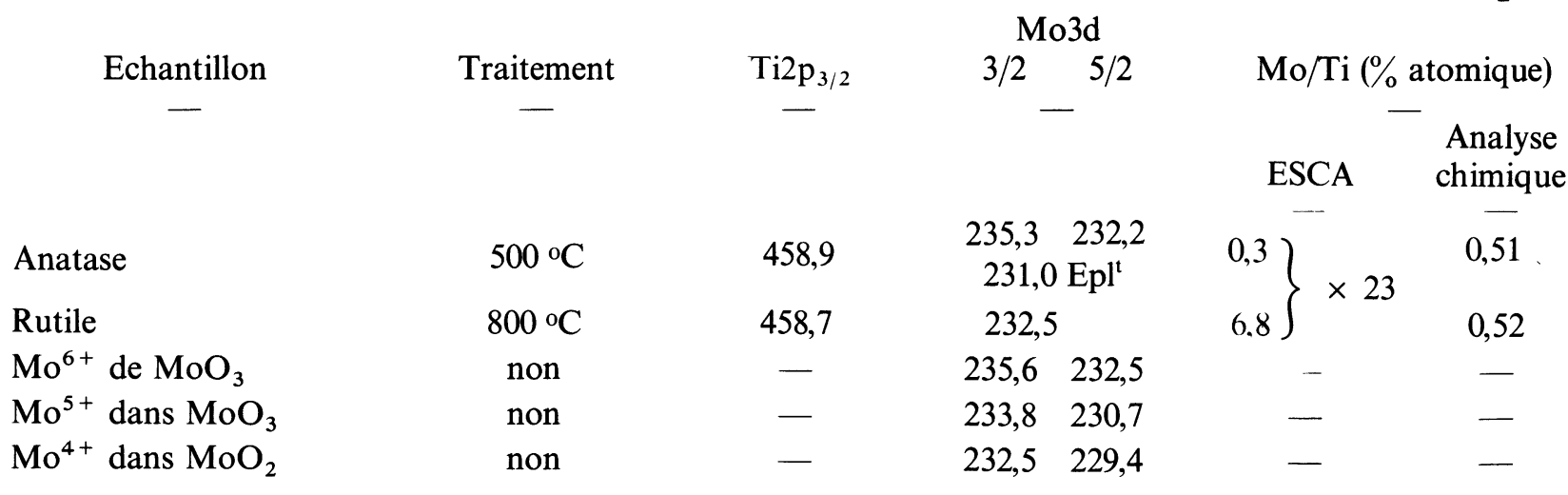


et de diffraction des rayons $\mathrm{X}$ [18] ont permis de suivre l'incorporation de $\mathrm{Sb}$ dans $\mathrm{SnO}_{2}$ avec formation de solution solide. Lorsque l'on introduit l'antimoine, il y a d'abord une insertion de $\mathrm{Sb}^{5+}$ avec un maximum de conductivité électrique pour une teneur de $6 \%$ en $\mathrm{Sb}$ puis de $\mathrm{Sb}^{3+}$. Enfin pour des teneurs élevées on observe un mélange de $\mathrm{Sb}_{2} \mathrm{O}_{4}$ et de solution solide.

Lorsque les solides précédents sont calcinés à des températures supérieures à $650^{\circ} \mathrm{C}$ on observe par ESCA que l'antimoine - au préalable régulièrement réparti entre la surface et le volume (en accord avec l'analyse chimique) - diffuse vers la surface des grains qui frittent fortement leur diamètre moyen passant grossièrement de 70 à $1000 \AA$ comme on l'a déterminé par microscopie électronique. Par diffraction des rayons $\mathrm{X}$ et pour des teneurs suffisantes en $\mathrm{Sb}_{2} \mathrm{O}_{4}$ ( $>10 \%$ ) on observe alors la formation de $\mathrm{Sb}_{2} \mathrm{O}_{4}-\alpha$ bidimensionnel. Le solide est alors composé de $\mathrm{Sb}_{2} \mathrm{O}_{4}$ à la surface de la solution solide de $\mathrm{Sb}$ dans $\mathrm{SnO}_{2}$. Lorsque la teneur en Sb est élevée ( $>40 \%$ ) on observe le phénomène inverse c'est-à-dire une diminution de la concentration superficielle en Sb par traitement thermique à haute température. Une étude parallèle de la sélectivité de ces solides dans la réaction d'oxydation ménagée du propène en acroléine a permis de suggérer que la phase active est vraisemblablement constituée de cet oxyde orienté $\mathrm{Sb}_{2} \mathrm{O}_{4}$ à la surface de la solution solide de $\mathrm{Sb}$ dans $\mathrm{SnO}_{2}$, cette sélectivité étant maximale lorsque l'on a ce dépôt bidimensionnel de $\mathrm{Sb}_{2} \mathrm{O}_{4}$.

6.3 INTERACTION MÉTAL-SUPPORT. - Cet aspect est important puisqu'il peut expliquer les propriétés particulières des métaux en fonction du support qui n'est plus, alors, totalement inerte. Un exemple est fourni par des catalyseurs de Pt sur zéolithe Y (silico-aluminate cristallisé). Après échange des ions $\mathrm{Na}^{+}$de la zéolithe de départ par des ions $\mathrm{Pt}^{2+}$ et réduction par l'hydrogène dans des conditions déterminées, on obtient des particules métalliques de diamètre 10 ou $25 \AA$ insérées dans les cavités de la zéolithe. Les propriétés catalytiques du Pt sont fortement modifiées par rapport au métal supporté sur silice ou alumine ce qui a été attribué à une forte interaction métal-support. Une telle interaction pou- vant induire des transferts électroniques métal-support nous avons effectué des études par ESCA. Nous avons observé [19] un déplacement important $(+0,8 \mathrm{eV})$ des raies du $\mathrm{Pt} 4 \mathrm{f}$ vers les énergies de liaison élevées ce qui correspond à un déficit électronique de $\mathrm{Pt}$, inférieur néanmoins à ce qu'il est pour $\mathrm{Pt}^{+1}$. Nous avons également étudié les propriétés d'accepteur et de donneur d'électron de la zéolithe par RPE, comme on l'a vu plus haut (\$3.2), à l'aide de pérylène et tetracyano éthylène, respectivement. Nous avons constaté un très grand accroissement des propriétés donneur d'électrons du support par un facteur de 10. L'utilisation des techniques de RPE et d'ESCA permet donc de conclure que la forte interaction métal-support induit un transfert électronique du métal vers le support ce qui a été confirmé par des mesures de seuil d'absorption de rayons $X$.

7. Conclusion. - Nous avons essayé tout au long de cet article de présenter les principaux aspects observés en catalyse et comment l'emploi de tout l'arsenal des méthodes physiques dont nous disposons peut permettre d'améliorer notre connaissance du catalyseur et de mieux sérier les nombreux problèmes qui se posent. On a vu que l'utilisation des techniques dans les conditions de la réaction demeure relativement limitée et que l'utilisation conjointe de plusieurs techniques, en particulier de surface et de volume, est indispensable pour une connaissance, approfondie et donc plus sûre des systèmes catalytiques.

Les progrès réalisés durant ces dix dernières années avec le développement ou l'amélioration des performances de nombreuses méthodes physiques ont permis de mieux appréhender les phénomènes d'adsorption et de phases actives. De grands progrès sont encore nécessaires pour déterminer la nature précise des sites catalytiques et des espèces intermédiaires. L'apport des méthodes physiques sera indispensable mais les méthodes classiques, en particulier de la chimie, ne doivent pas être négligées pour autant. Nous dirons aussi pour conclure que la réaction catalytique demeure encore le meilleur test des propriétés superficielles et que la diversification de ces réactions et des réactifs peut amener des informations très importantes.

\section{Bibliographie}

[1] Mentzen, B., Spectroscopies infra rouge et raman. Monographies du CAST. (Masson et Cie. Paris) 1974.

KLIER, K., Reflectance spectroscopy as a tool for investigating dispersed solids and their surfaces. Catal. Rev. 1 (1968) 207.

GeORGE, W. O., Spectroscopic methods in organo metallic chemistry, Butterworth, Londres (1970).

[2] Hugues, T. R. et White, J. Phys. Chem. 71 (1967) 2192.

[3] Foster, D., J. Am. Chem. Soc. 98 (1976) 846 et Adv. Organomet. Chem. 17 (1979) 255.

[4] RAo, C. N. R., Ultraviolet and visible'spectroscopy : Chemical applications. Butterworths, Londres (1970).

[5] Freymann, R. et Soutif, M., La spectroscopie hertzienne appliquée à la Chimie (Dunod. Parìs) 1960 ;
Abragam, A. et Bleaney, B., RPE des ions de transition (P.U.F., Paris) 1971 ;

URSU, I., La résonance paramagnétique électronique (Dunod, Paris) 1968 ;

Derouane, E. G., Fraissard, J., Fripiat, J. J. et Stone, W. E., NMR Studies in adsorption and heterogeneous catalysis. Catal. Rev. 7 (1972) 121 ;

Che, M., Fraissard, J. et Vedrine, J. C. Applications de la RMN et de la RPE à l'étude des silicates et des argiles. Bull. Groupe Fr. Argiles 26 (1974) 1.

[6] HerPin, A., Théorie du magnétisme (P.U.F., Paris) 1968. Selwood, P. W., Chemisorption et magnetization (Academic Press. London) 1975. 
[7] JANOT, C. L'effet Mössbauer et ses applications à la physique du solide et à la métallurgie physique (Masson et Cie, Paris) 1972.

Dumescic et TopsoE. Catalyse heterogène. Adv. Catal. 26 (1976) 121.

[8] Magnan, C., Traité de microscopie électronique (Edit. Hermann, Paris) 1961.

Eberhart, J. P., Méthodes physiques d'étude des minéraux et des matériaux solides (Doin Editeurs) 1976.

[9] Par exemple : Delannay, F., Catal. Rev. 22 (1980) 141.

[10] GuINIER, A., La diffraction des rayons $X$. Théorie et technique de la radiocristallographie (Dunod, Paris) 1956.

[11] Azaroff, L. Y., $X$ rays spectroscopy. (Mc Graw Mill, New York) 1976.

STERn, E. A., EXAFS and its applications to surface structure J. Vac. Sci. Technol. 14 (1977) 461 ;

Raoux, D., Petiau, J., Bondot, P., Calas, G., Fontaine, A. Lagarde, P., Levitz, P., Loupias, G. et Sadoc, A., Revue Phys. Appl. 15 (1980) 1079.

[12] Gray, T. J. dans The defect solid state (edited by Intersc. Publish., New-York) 1975, chapitres p. 25, 57, 77 et 239.

[13] BRUndle, C. R. et BAKER, A. D., Electron spectroscopy : theory, techniques and applications (Academic Press, Londres) 1977.

VÉDRINE, J. C. La spectroscopie d'électrons appliquée à l'étude des catalyseurs et des phases adsorbées. J. Microsc. Spectrosc. Electron. 1 (1976) 285.
[14] Benninghoven, A., Surf. Sci. 53 (1975) 596.

WERner, H. W., Surf. Sci. 47 (1975) 301.

Par exemple : Delannay, F., Haeussler, E. N. et Delmon, B., J. Catal. 66 (1980) 469.

[15] Meriaudeau, P. et Védrine, J. C., Nouv. J. Chim. 2 (1978) 133. Védrine, J. C., Praliaud, H., Meriaudeau, P. et Che, M., Surf, Sci. 80 (1979) 101.

[16] Boudeville, Y., Figueras, F., Forissier, H., Portefaix, J. L. et Védrine, J. C., J. Catal. 58 (1979) 52.

[17] Herrmann, J. M. et Portefaix, J. L., React. Kinet. Letters 12 (1979) 51.

[18] Portefaix, J. L., Bussiere, P., Figueras, F., Forissier, H., Friedt, J. M., Theotald, F. et Sanchez, J. P., Faraday Trans. I. 76 (1980) 1652.

[19] Védrine, J. C., Dufaux, M., Naccache, C. et Imelik, B., J. Chem. Soc. Faraday Trans. I 74 (1978) 440.

[20] Lyon, Institut de Recherches sur la Catalyse, CNRS, F69626 Villeurbanne. Liste des conférences présentées (sujet : surfaces, grains fins, catalyse).

Dormann, J. L., à paraître dans la Revue Phys. Appl.

Bussiere, P., à paraître (Rev. Phys. Appl.).

Mathieu, M. V., à paraître (Rev. Phys. Appl.).

Martin, G. A., à paraître dans la Revue Phys. Appl. 